\title{
EFFECT OF DEFORMATION ON POSITRON ANNIHILATION IN DECOMPOSING COPPER ALLOYS
}

\author{
V.S. Mikhalenkov \\ Institute of Metal Physics, Ukrainian Academy of Sciences, 262680 Kiev-142, Ukraine \\ P. HaUtojärvi AND A. Vehanen
}

Laboratory of Physics, Helsinki University of Technology, 02150 Espoo-15, Finland

Positron lifetime spectra were measured in the quenched or slowly cooled $\mathrm{Cu}-3.93$ at.\% Ag alloy, subjected to subsequent cold rolling, as a function of isochronal annealing temperature. The results were compared with the formerly obtained data for quenched, but not deformed, $\mathrm{Cu}-\mathrm{Ag}$ alloy and for deformed $\underline{\mathrm{Cu}}-\mathrm{Ge}$ solid solution. All lifetime spectra, after background and source corrections, are single exponential with the lifetimes which exceed those in well-annealed $\mathrm{Cu}$ solid solutions. This gives evidence to positron annihilation from a trapped state. One-component spectra may then testify to existence of a set of different traps with too weak distinction in the individual positron lifetime to be resolved as separate components. At low annealing temperatures the stage arisen from continuous decomposition is not revealed, being masked by the removal of deformation defects. The stages of discontinuous decomposition and of subsequent precipitates coagulation turn out to depend on the sample history. The role of deformation defects in the decomposition of supersaturated solid solutions is attempted to be accounted for in a wide range of annealing temperatures.

PACS numbers: 61.66.Dk, 61.72.Ff, 78.70.Bj, 81.30.Mh

\section{Introduction}

Crystalline structure defects exert essential influence on the decomposition of supersaturated solid solutions. Their effect is especially strong in systems, where specific volume of precipitates exceeds considerably that of solid solution. In this case the precipitates are able to grow incorporating vacancies, which provide relaxation of elastic stresses arisen at the precipitate boundary. $\mathrm{Cu}-\mathrm{Ag}$ alloys belong to such systems since the specific volume of $\alpha_{\mathrm{Ag}}$-phase, which is formed in the course of decomposition of the supersaturated solid solution, is $13.5 \%$ larger than that of $\mathrm{Cu}-\mathrm{Ag}$ matrix. During annealing of the quenched $\underline{\mathrm{Cu}}-\mathrm{Ag}$ alloy both continuous and discontinuous mechanisms of decomposition can be realised [1].

In the preceding paper [2] the stage of continuous decomposition in the quenched $\mathrm{Cu}-3.93$ at.\% Ag alloy was shown to be accompanied by formation of 
clusters consisting of 2-4 vacancies. These clusters were suggested to compensate the surplus volume of precipitates and to promote their growth. The exhaustion of vacancy excess calls forth the change in decomposition mechanism and gives rise to the formation of discontinuous precipitate colonies which appear at the grains and phases boundaries $[1,3]$.

Dislocations and vacancy-type defects introduced by plastic deformation must affect the decomposition processès. Really, deformation of quenched $\mathrm{Cu}-4.17$ at.\% $\mathrm{Ag}$ alloy was found out to change the ratio between continuous and discontinuous precipitates formed during subsequent ageing $[4,5]$. The rate of discontinuous precipitation depends on the degree of deformation. The larger is deformation degree, the higher are the growth rate of cellular precipitate colonies and the probability of secondary coarse-lamellar precipitate formation. In this paper the effect of preliminary deformation on the behaviour of defects in the course of decomposition in $\mathrm{Cu}-3.93$ at.\% $\mathrm{Ag}$ alloy is studied by means of positron lifetime (PLT) spectroscopy.

\section{- 2. Experimental}

The alloy was prepared from the constituent metals of $4 \mathrm{~N}$ purity by arc-melting in vacuum. The composition was verified by chemical analysis. After homogenization for $48 \mathrm{~h}$ at $1125 \mathrm{~K}$ the ingot was cold rolled into a strip of $1.2 \mathrm{~mm}$ thickness. Each sample for PLT measurements consisted of two identical plates, $10 \times 10 \mathrm{~mm}$ in size, cut out of the strip. Two such couples, after being kept for $0.5 \mathrm{~h}$ at $1090 \mathrm{~K}$ in purified $\mathrm{Ar}$ atmosphere, were cooled under different conditions. One of them, 1 , was quenched into water, whereas the other, 2 , was moved into the unheated part of the furnace and underwent natural cooling. The final treatment of both samples was cold rolling to $60 \%$ thickness reduction. Thus, the initial state of the sample 1 represented the supersaturated solid solution, whereas the sample 2 contained, besides deformation defects, continuous precipitates of $\alpha_{\mathrm{Ag}}$-phase formed during cooling.

In the course of investigation the samples were subjected to the stepped isochronal annealing in vacuum in the range from room temperature up to $975 \mathrm{~K}$. The duration of each step was $0.25 \mathrm{~h}$. After each annealing PLT spectrum was measured at room temperature using conventional fast-slow coincidence system with $270 \mathrm{ps}$ time resolution [6]. The ${ }^{22} \mathrm{NaCl}$ positron source of $15 \mu \mathrm{Ci}$ activity wrapped into thin $\left(1.1 \mathrm{mg} \mathrm{cm}^{-2}\right) \mathrm{Ni}$ foil was sandwiched between the plates. $5 \times 10^{5}$ coincident events were collected in each experiment. The PLT spectra were analysed using elaborated in Helsinki University of Technology POSLIFFIT programme which provided, after background and source correction, for two-component decomposition. However, for all treatments of the samples in question the best fit was achieved by representing the spectrum with one exponential component.

\section{Results}

The obtained dependences of PLT on the isochronal annealing temperature, $T_{\text {an }}$, are shown in Fig. 1. Formerly obtained results for the quenched, but not deformed, $\mathrm{Cu}-3.93$ at.\% $\mathrm{Ag}$ alloy [2] and for deformed $\mathrm{Cu}-5$ at.\% Ge solid solution [7] are plotted in the same figure for comparison. 


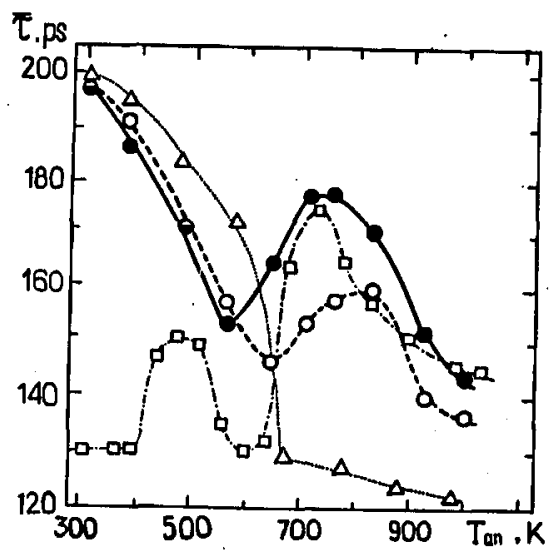

Fig. 1. Mean positron lifetime in the quenched (squares), deformed after quenching (open circles) and after slow cooling down (filled circles) $\mathrm{Cu}-3.93$ at.\% $\mathrm{Ag}$ alloy as a function of annealing temperature. The data for $\mathrm{Cu}-5$ at. $\%$ Ge solid solution (triangles) taken from Ref. [7] are displayed for comparison. The lines are the guides to an eye.

Apart from some distinction in the levels and positions of the observed peculiarities, the general view of PLT dependence on $T_{\text {an }}$ for the samples 1 and 2 is rather similar. Initial PLT in the as-deformed samples 1 and 2 amounts to $197 \mathrm{ps.}$ This value is close to PLT in deformed $\mathrm{Cu}$ and $\mathrm{Cu}-5$ at.\% Ge alloy. Insensitivity of PLT in deformed Cu alloys to the preliminary heat treatment stands for the preferential positron trapping at the deformation induced defects, i.e. vacancies and dislocations.

In contrast to the quenched sample, the hump of PLT, observed at $T_{\text {an }}$ ranged between $400 \mathrm{~K}$ and $600 \mathrm{~K}$ and attributed to the continuous decomposition, is not revealed in deformed samples 1 and 2 . However, the absence of PLT maximum at $T_{\text {an }}=500 \mathrm{~K}$ does not mean that the continuous decomposition does not occur in these samples. More likely that the increase in PLT due to formation of continuous precipitates is masked by such recovery processes in a deformed alloy as moving out deformation defects, formation of vacancy-impurity complexes, decoration of dislocations with impurity atoms etc., which are known to reduce PLT.

According to Ref. [8], strong (more than 50\% thickness reduction) deformation, though brings down the contribution of continuous precipitates in $\mathrm{Cu}-\mathrm{Ag}$, does not suppress their formation totally. More rapid drop of PLT at $T_{\text {an }}<600 \mathrm{~K}$ in the sample 1 , as compared with slowly cooled sample 2 , may be considered as an indirect evidence to development of continuous decomposition in the former object. The embryos of $\alpha_{\mathrm{Ag}}$-phase are effective sinks for vacancies as they are required for the precipitates growth.

After a fall down, caused by moving out deformation defects, PLT begins to rise in both 1 and 2 samples approaching maxima. However, the value and position of these maxima, as well as the course of the subsequent PLT decrease, are different for these samples. 


\section{Discussion}

The main feature of all measured PLT spectra is their one-component fit. At the same time PLTs in all cases turn out to exceed those typical for annihilation in perfect lattice of both $\mathrm{Cu}$ and $\mathrm{Ag}$ (about $120 \mathrm{ps}$ ). This fact gives evidence to the annihilation of, at least, a part of positrons from the trapped state. The one-component fit may then testify to the existence of a set of positron traps with too weak distinction in the individual PLT to be resolved as the separate components in the total spectrum. The possible candidates for these traps are vacancies and their complexes with $\mathrm{Ag}$ atoms, clean and decorated by $\mathrm{Ag}$ atoms dislocations, continuous and discontinuous precipitates and accommodation defects at their boundaries. Positron trapping at the grain boundaries is, in principle, also possible but, taking into account big grain size (several tens of $\mu \mathrm{m}$ ), the contribution of this mechanism is suggested to be insignificant. The estimation, based on data given in Ref. [9], shows that, owing to a-difference in positron affinity to $\mathrm{Cu}$ and $\mathrm{Ag}$ atoms, the segregation of $\mathrm{Ag}$ more than $4 \mathrm{~nm}$ in radius are able to localize the positron. This gives proof of positron trapping inside the precipitates.

The absence of long-lived PLT component is evidence that vacancy clusters, observed in quenched $\mathrm{Cu}-\mathrm{Ag}$. alloy [2], are not formed in deformed samples. It was suggested in Ref. [2] that such clusters provide accommodation of continuous $\alpha_{\mathrm{Ag}}$-phase precipitates. Probably, deformation defects, stabilized by Ag atoms, promote continuous precipitates growth in the deformed alloy, as vacancy clusters do in the quenched one. The vacancy-impurity complexes formation is favoured by the fact that the atomic size of $\mathrm{Ag}$ is by $12 \%$ larger than that of $\mathrm{Cu}[10]$.

More sharp drop of PLT at low $T_{\text {an }}$ in $\mathrm{Cu}-\mathrm{Ag}$ with respect to $\mathrm{Cu}-\mathrm{Ge}$ (Fig. 1) enables one to conclude that deformation defects in decomposing alloy move out faster than in a solid solution. The reason for this effect may consist in higher concentration of sinks in the former object. The embryos of new phase may serve as defect sinks - the embryos may consume vacancies when the embryos grow.

The change of character of PLT dependence on $T_{\mathrm{an}}$ at $T_{\mathrm{an}}>600 \mathrm{~K}$ gives evidence that some new processes are involved into PLT variation. From analogy with results for undeformed $\mathrm{Cu}-\mathrm{Ag}$ alloy [2] one can accept that this change is a consequence of discontinuous precipitate formation. The beginning of this stage in the sample 1 is shifted to lower $T_{\text {an }}$, with respect to both sample 2 and the quenched alloy. Such behaviour is in agreement with the results of $[4,5]$ and indicates that deformation defects promote discontinuous precipitates nucleation. In particular, nucleation of these precipitates may occur not only at the grain boundaries but at the dislocation segregation inside the grains, too. Thus the rise of PLT at $T_{\mathrm{an}}>600 \mathrm{~K}$ should be identified with positron trapping at defects which are formed during discontinuous precipitation. It is worth reminding that discontinuous precipitates growth proceeds in collaboration with the climb up of partial dislocations which emit vacancies necessary to compensate surplus volume conditioned by $\alpha_{\mathrm{Ag}}$-phase $[1,3]$. One may consider these vacancies to be of "dynamic" nature as once being sprung up at the dislocations they are consumed quite soon at the boundaries of precipitates. Nevertheless, at every moment of the precipitates growth there is an excess of vacancies that move from the source to 
the sink. The rise in vacancy concentration at the expense of growing precipitates surface is suggested to be responsible for the increase in PLT at $T_{\text {an }}>600 \mathrm{~K}$. However, the rate of PLT increase and PLT maximum value turn out to depend on the sample history. In the quenched sample and in the deformed sample 1 the highest PLT at this stage is attained at $T_{\text {an }}$ about $720 \mathrm{~K}$ and amounts to $175 \mathrm{ps}$. This means that at such high $T_{\text {an }}$ the deformation defects do not affect any more on the precipitate growth. Assuming that, in both quenched sample and deformed sample 1, the positron traps are vacancy-like defects, one may conclude that, after annealing at $720 \mathrm{~K}$, their concentrations are about the same in both samples. Thus, after the deformation defects are exhausted, the discontinuous precipitation process proceeds similarly in these samples. But this does not mean at all that the size and distribution of precipitates are identical in both samples after final treatment.

In the sample 2, slowly cooled before deformation, the dependence of PLT on $T_{\text {an }}$ looks different. The maximum of PLT is attained at higher $T_{\text {an }}$ (about $800 \mathrm{~K}$ ) and amounts to $160 \mathrm{ps}$ only, indicating on the reduced concentration of positron traps. This fact may be accounted for by the decrease in Ag concentration in solid solution at the expense of $\alpha_{\mathrm{Ag}}$-phase formed during the slow cooling down. Therefore the subsequent decomposition, being limited by diffusion of $\mathrm{Ag}$ atoms to the precipitate embryos, proceeds at a lower rate. This leads to the reduced density of defects drown into precipitates growth process and results in the decrease in positron traps concentration.

PLT decrease in the quenched $\mathrm{Cu}-\mathrm{Ag}$ at $T_{\text {an }}>720 \mathrm{~K}$ was attributed in Ref. [2] to the coagulation of precipitates accompanied by reduction in the interphase boundary length. From this viewpoint, more sharp PLT drop in the quenched sample stands for more rapid completion of coagulation process, as compared with the deformed sample 1. However the lowest PLT level (145 ps) attained in both samples is the same and exceeds PLT in well-annealed both pure $\mathrm{Cu}$ and $\mathrm{Cu}-5$ at.\% Ge alloy. Thus, in the decomposing $\mathrm{Cu}-\mathrm{Ag}$ alloy the positron traps are still retained after $975 \mathrm{~K}$ anneal. The candidates for these traps are defects at the boundaries of precipitates.

In the sample 2 the $975 \mathrm{~K}$ anneal results in PLT decrease to $135 \mathrm{ps}$, i.e. to the value that is higher than in well-annealed $\mathrm{Cu}$ but lower than in the sample 1 . The positron traps in this case must be also defects at the boundaries of precipitates. But the early formation of the precipitates during the sample cooling down results finally in more coarse precipitates with the lesser total length of the boundaries.

In conclusion, the positron lifetime study of deformed $\mathrm{Cu}-3.93$ at.\% $\mathrm{Ag}$ alloy provided information about positron interaction with the defects arisen at different stages of decomposition process. The role of deformation defects in the precipitate formation is attempted to be cleared up.

\section{References}

[1] R. Räti, H.M. Miekk-oja, Phylos. Mag. 18, 1105 (1968).

[2] V.S. Mikhalenkov, P. Hautojärvi, A. Vehanen, Ukrainian Phys. J. 29, 1048 (1984) (in Russian). 
[3] R. Wirth, H. Gleiter, J. Mater. Sci. 16, 557 (1981).

[4] W. Scharfenberger, G. Schmidt, H. Borchers, Z. Met. 63, 553 (1968).

[5] W. Leo, Z. Met. 58, 456 (1967).

[6] Positrons in Solids, Ed. P. Hautojärvi, Springer-Verlag, Berlin 1979.

[7] P. Hautojärvi, A. Vehanen, V.S. Mikhalenkov, Solid State Commun. 19, 309 (1976).

[8] H. Borchers, W. Scharfenberger, R. Steege, Metall. 22, 405 (1968).

[9] M.J. Puska, P. Lanki, R.M. Nieminen, J. Phys., Condens. Matter 1, 6081 (1989).

[10] E.B. Royce, Phys. Rev. 164, 929 (1967). 